\title{
Trading of Intangible Goods
}

\author{
Dimitri Konstantas / Universty of Twente, Enschede, The Netherlandsdimitri@cs.utwente.nl \\ Erich Neuhold / Fraunhofer- IPSI, Darmstadt, Germany-neuhold@darmstadt.gmd.de \\ Vaggelis Ouzounis / Fraunhofer- FOKUS, Berlin, Germany- ouzounis@fokus.gmd.de \\ Fred Wall / HM Customs \& Excise, London, United Kingdomfred.wall@hmce.gov.uk
}

The evolution of different technologies, the high availability of robust networks and the ever increasing need for fast, reliable and low cost services and information have contributed in the wide acceptance and use of digital information and services, which are now days termed as Intangible Goods. Commercial companies and public administration are introducing every day new services and information in diital formats, while the network has become the first choice of users when looking for everyday services and information. As a result Electronic Intangible Goods are now part of every-day life.

This year the minitrack presents four papers discussing issues in the complete range of the Intangible Goods trading process, ranging from security issues, to authentication and platform requirements.

The first paper in this minitrack, Privacy in Distributed Electronic Commerce, by Lary Korba, describes the issues that provoke privacy challenges for agent-based e-commerce due to current and impending privacy legislation as well as an approach for policy driven privacy negotiation for use in distributed agent based systems. The wide acceptance and use of Intangible Goods can be sustained only if both providers and users are assured of their reliability, security, and robustness. Users need to be assured that the use of electronic services and access of electronic information will not jeopardize their privacy. On the other hand maintaining the user privacy should be done in a way that is compatible with existing legislation in national and international level.

The second paper, Information Hiding with a Handwritten Message in Vectordrawing Codes by Norihisa Segawa, Yuko Murayama and Masatoshi Miyazaki, presents some new algorithms for information hiding with a handwritten message that is encoded with vector drawing, providing a subliminal communication channel. In handwritten documents the author can be easily identified by his handwriting. When PDAs are used for the exchange of messages in bulleting boards, as is the case in Japan, a user might need, from one hand to hide his identity when publishing in a public channel, and on the other hand provide information to knowledgeable users which can act as a security safeguard, using a subliminal channel.

The third paper, Hermes - A Lean M-Commerce Software Platform Utilizing Electronic Signatures by Sebastian Fischmeister and Wolfgang Pree, presents the Hermes platform, a lean yet secure trading platform that utilizes digital signatures for authentication that is compliant with the Austrian electroniesignature law. Of major importance in ecommerce is the ability of the participants to provide evidence in court about the validity of contracts and transactions. In traditional commerce, this is done by the handwritten signature of the parties on the contract. In e-commerce digital signatures provide the equivalent of handwritten signatures. However existing systems utilizingelectronic signatures are too heavy for the emerging mobile commerce (m-commerce) applications

The last paper, FAIRWIS: An Integrated System Offering Trade Fair Web-based Information Services A $R \& D$ Case Study by Claudio Muscogiuri, Gerald Jäschke, Aldo Paradiso and Matthias Hemmje describes as a case study the FAIRWIS system, an integrated system offering trade-fair Web-based information. When all security problems are takes care off, the users and the service and information providers will need system that allow them to interact with each other, and platforms where new services can be introduced easily and fast. This requires frameworks and integrated computational environments able to model, develop and support business processes in all their steps. 